(HR 1.619, $\mathrm{p}=0.018$ ) and uveitis ( $H R$ 1.732, $\mathrm{p}=0.025$ ) as predictors for worse drug survival. At the time of censoring only $202(11.6 \%)$ patients were using csDMARDs with TNFi agent. When we repeat the analysis with patients continuing csDMARDs we showed that there is no significant differences regarding TNFi survival.

Conclusions: The results of present study showed that considerable amount of SpA patients were using csDMARDs at time of first TNFi initiating and drug survival was significantly better in those patients.

Disclosure of Interest: None declared

DOI: 10.1136/annrheumdis-2018-eular.4646

\title{
SAT0298 TRANSCUTANEOUS VAGUS NERVE STIMULATION IN PATIENTS WITH PSORIATIC ARTHRITIS OR ANKYLOSING SPONDYLITIS
}

S.E. Rasmussen ${ }^{1}$, C. Brock ${ }^{1,2}$, A. Mohr Drewes ${ }^{1}$, M. Pfeiffer Jensen ${ }^{1} .{ }^{1}$ Department of Rheumatology, Aarhus University Hospital, Aarhus $C ;{ }^{2}$ Mech-Sense, Department of Medical Gastroenterology, Aalborg University Hospital, Aalborg, Denmark

Background: Psoriatic arthritis and ankylosing spondylitis are chronic autoimmune diseases characterised by inflammation of both peripheral and spina joints. The chronic inflammation may lead to impaired functionality and absenteeism from work. It has become increasingly accepted that the vagus nerve plays a pivotal role as a communicator between the immune and nervous systems ${ }^{1}$. Recent studies in similar immune-mediated inflammatory diseases have demonstrated promising anti-inflammatory effect of vagus nerve stimulation ${ }^{2,3}$

Objectives: We hypothesised that transcutaneous vagus nerve stimulation (tVNS) would increase the activity of the vagus nerve, and that this enhanced parasympathetic tone would reduce inflammation and disease activity. The aims of this study were to investigate the effects of t-VNS on cardiac vagal tone and disease activity in patients with psoriatic arthritis or ankylosing spondylitis.

Methods: Twenty patients diagnosed with psoriatic arthritis (median disease duration 7.0 years) and 17 patients diagnosed with ankylosing spondylitis (median disease duration 4.5 years) were included in the study. Transcutaneous vagus nerve stimulation for $120 \mathrm{~s}$ was performed bilaterally, thrice daily for four days, using a non-invasive handheld device. Cardiac vagal tone, measured on a linear vagal scale (LVS), and disease activity scores were assessed on the 1 st, 2nd and 5 th day.

Results: In the ankylosing spondylitis group, cardiac vagal tone was significantly increased $30 \mathrm{~min}$ after t-VNS in comparison to baseline (6.46 vs. 6.57. LVS, $\mathrm{p}<0.05$ ) (figure $1 \mathrm{~A})$, accompanied by a significant reduction in heart rate $(68.44$ vs. $65.37, \mathrm{p}<0.01)$. Transcutaneous vagus nerve stimulation did not change cardiac vagal tone in the psoriatic arthritis group (figure $1 \mathrm{C}$ ), however, a significant reduction in heart rate $30 \mathrm{~min}$ after t-VNS in comparison to baseline was observed (71.26 vs. 68.04, $\mathrm{p}<0.01$ ). A significant reduction in ASDAS score was demonstrated in the psoriatic arthritis group on both day $2(2.09$ vs. $1.96, p<0.05)$ and day 5 (2.09 vs. $1.88, p<0.01$ ) (figure 1D)

$A_{20.0}$

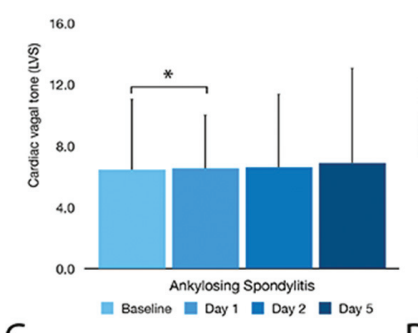

C

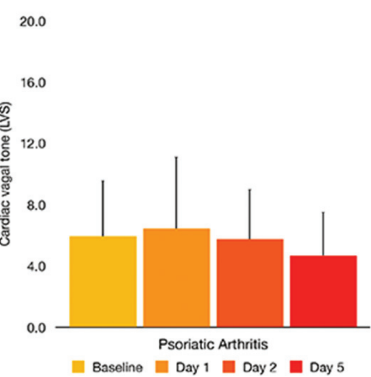

B 5.00

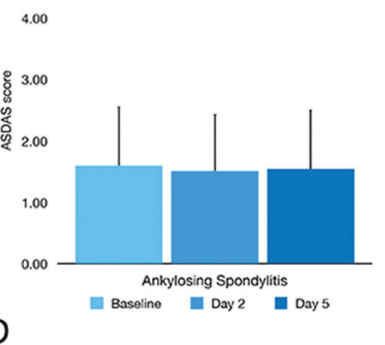

5.00

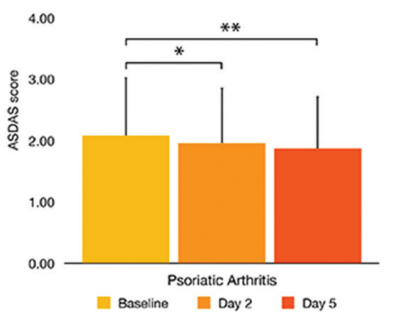

Conclusions: Transcutaneous vagus nerve stimulation may have an acute effect on cardiac vagal tone and heart rate in patients with ankylosing spondylitis and psoriatic arthritis. The observed effect of t-VNS on ASDAS scores in patients with psoriatic arthritis was not associated with a modulation of cardiac vagal tone.

\section{REFERENCES:}

[1] Rasmussen S, et al. Vagal influences in rheumatoid arthritis. Scand. J. Rheumatol 2018:47:1-11.

[2] Koopman FA, et al. Vagus nerve stimulation inhibits cytokine production and attenuates disease severity in rheumatoid arthritis. Proc. Natl. Acad. Sci 2016;113:8284-8289.

[3] Bonaz B, et al. Chronic vagus nerve stimulation in Crohn's disease: a 6month follow-up pilot study. Neurogastroenterol. Motil 2016;28:948-953.

Acknowledgements: This project was supported by the Danish Psoriasis Research Fund and the Danish Rheumatism Association.

Disclosure of Interest: None declared

DOI: 10.1136/annrheumdis-2018-eular.4731

\section{SAT0299 REAL WORLD SECUKINUMAB STUDY IN AS AND PSA - COMORBIDITIES AND EXTRAARTICULAR MANIFESTATIONS: INCIDENCE AND STATUS THROUGHOUT NON-INTERVENTIONAL AQUILA STUDY IN GERMANY}

U. Kiltz ${ }^{1}$, P. Kästner ${ }^{2}$, H. Krauel ${ }^{2}$, I. Schwarze ${ }^{3}$, J. Brandt-Jürgens ${ }^{4}$, M. MaierPeuschel $^{5}$, C. Legeler ${ }^{5}$, J. Veit ${ }^{5}$, H.-P. Tony ${ }^{6}$ on behalf of the AQUILA Study Group.

${ }^{1}$ Rheumazentrum Ruhrgebiet, Herne; ${ }^{2}$ Ambulantes Rheumazentrum, Erfurt,

${ }^{3}$ Praxis für internistische Rheumatologie, Leipzig; ${ }^{4}$ Rheumatologische Schwerpunktpraxis, Berlin; ${ }^{5}$ Novartis Pharma GmbH, Nürnberg; ${ }^{6}$ Medizinische Klinik II - Rheumatologie/Immunologie, Universitätsklinikum Würzburg, Würzburg, Germany

Background: Patients with psoriatic arthritis (PsA) or ankylosing spondylitis (AS) may suffer from extraarticular manifestations (EAM) (uveitis, psoriasis) and have higher rates of comorbidities like cardiovascular diseases (CVD) or depression than the normal population. Comorbidities should be kept in mind when managing pts with PsA and AS, as they can contribute to increased mortality and influence disease activity ${ }^{1}$.

Objectives: The aim of this interim analysis is to evaluate the incidence of selected EAM and comorbidities at baseline and the impact of secukinumab on the status of these attributes compared to baseline.

Methods: The presence and severity of uveitis and psoriasis (assessed by PASI) as well as diagnosis of coronary heart disease (CHD), stroke, heart insufficiency, and depression (assessed by Becks Depression Inventory (BDI-II) were docu mented according to clinical routine at baseline and at week 4, 16, 24 and 52 after initiation of secukinumab. At baseline 486 patients were included and observed up to 52 weeks, at the time of analysis not all patients have already reached the end of the study, therefore the results are presented as observed.

Results: As expected, current plaque psoriasis was very frequent in PsA pts $(63.3 \%)$, but also present in AS pts $(11.5 \%)$ at baseline. For PsA pts, median PASI improved from 5.0 at baseline to 0.0 at wk 52 . Half of pts with available PASI achieved clear skin at wk 52 (Tab 1)

Uveitis was more frequent in AS pts than in PsA pts (6.2\% vs $0.9 \%)$. Only 1 AS pt and 2 PsA pts experienced new onset of uveitis.

CVD were more frequent in PsA than in AS patients at baseline: $\mathrm{CHD}$ (PsA 8.9\%, AS 3.5\%), heart failure (PsA 3.3\%, AS 0.7\%) and stroke (PsA $2.4 \%$, AS $0.0 \%$ ). During 52 wks study duration none comorbidity worsened with secukinumab treat ment. Two patients with PsA were newly diagnosed with $\mathrm{CHD}$ and heart insufficiency throughout wk 52. No new stroke occurred.

Depression was common in both populations (PsA 15.4\%, AS 12.2\%) at baseline. Up to wk 52 median BDI-II improved from 12.0 to 6.0 (AS pts) and from 9.0 to 6.0 (PsA pts [Tab 1]).

Abstract SAT0299 - Table 1. PASI and BDI response under secukinumab

\begin{tabular}{lcccccc}
\hline & Baseline & Wk 4 & Wk 16 & Wk 28 & Wk 40 & Wk 52 \\
\hline PASI 100 n(\%) & $0(0.0)$ & 7 & 23 & 28 & 18 & 15 \\
& & $(13.0)$ & $(34.3)$ & $(47.5)$ & $(41.9)$ & $(50.0)$ \\
PASI 75 n(\%) & $0(0.0)$ & 9 & 26 & 33 & 21 & 18 \\
& & $(16.7)$ & $(38.8)$ & $(55.9)$ & $(48.8)$ & $(60.0)$ \\
BDI-II (AS pts) & 12.0 & 9.0 & 8.0 & 8.0 & 7.5 & 6.0 \\
BDI-II (PsA & 9.0 & 8.0 & 7.5 & 8.0 & 7.0 & 6.0 \\
pts) & & & & & &
\end{tabular}


Conclusions: Incidence of CVD and depression in PsA and AS pts is generally comparable to the published literature ${ }^{2,3}$. However, in contrast to other studies ${ }^{4}$, previous uveitis was less frequently reported in $\mathrm{SpA}$ pts selected for treatment with a IL-17 inhibitor, particularly in the AS group. Cardiovascular comorbidities remained overall stable under secukinumab up to wk 52 . Plaque psoriasis and depressive mood improved with Secukinumab treatment.

\section{REFERENCES:}

[1] Tournadre A, et al. Ther Adv Musculoskel Dis 2016;8(5):180-91.

[2] Wibetoe G, et al. Arthritis Res Ther 2017;19(1):153.

[3] Husni ME, et al. Semin Arthritis Rheum 2017;47(3):351-60.

[4] Rosenbaum JT. Clin Rheumatol 2015;34:999-1002.

Acknowledgements: C. Blank, PhD (Winicker-Norimed) for medical writing support, which was funded by Novartis Pharma GmbH, Germany

Disclosure of Interest: U. Kiltz Grant/research support from: AbbVie, Chugai, Grünenthal, MSD, Novartis, Pfizer, Roche, UCB, Consultant for: AbbVie, Chugai, MSD, Novartis, Pfizer, Roche, UCB, P. Kästner Consultant for: Chugai, Novartis, H. Krauel: None declared, I. Schwarze: None declared, J. Brandt-Jürgens: None declared, M. Maier-Peuschel Employee of: Novartis Pharma GmbH, C. Legeler Employee of: Novartis Pharma GmbH, J. Veit Employee of: Novartis Pharma $\mathrm{GmbH}$, H.-P. Tony Consultant for: AbbVie, Astra-Zeneca, BMS, Chugai, Janssen, Lilly, MSD, Novartis, Pfizer, Roche, Sanofi

DOI: 10.1136/annrheumdis-2018-eular.1180

\section{SAT0300 TREATMENT EXPERIENCE AND SATISFACTION IN ANKYLOSING SPONDYLITIS PATIENTS TREATED WITH SECUKINUMAB: RESULTS FROM A US WEB-BASED SURVEY}

M. Magrey ${ }^{1}$, M. Bozyczko ${ }^{2}$, D. Wolin ${ }^{3}$, M. Mordin ${ }^{3}$, L. Mcleod ${ }^{3}$, E. Davenport ${ }^{3}$, Y. Park ${ }^{4} .{ }^{1}$ Case Western Reserve University, Cleveland, $\mathrm{OH}^{2}{ }^{2}$ National Psoriasis Foundation, Portland, OR; ${ }^{3} R T I$ Health Solutions, Research Triangle Park, NC; ${ }^{4}$ Novartis Pharmaceuticals Corporation, East Hanover, NJ, USA

Background: Secukinumab is the only interleukin-17A inhibitor approved for the treatment of ankylosing spondylitis (AS). There are limited real-world data about treatment experience and patient satisfaction with secukinumab among patients with AS.

Objectives: To evaluate real-world treatment experience and satisfaction with secukinumab in US patients with AS.

Methods: Data on demographics, AS symptoms, treatment history, and treatment satisfaction were collected from a cross-sectional, web-based panel survey from July 10 to August 3,2017. A random sample of US patients were invited to participate in the survey by Survey Sampling International via their patient panels. Eligibility criteria included patients aged $>18$ years with a self-reported diagnosis of AS who initiated secukinumab $>3$ months before participation and received secukinumab continuously since initiation. The outcomes evaluated were AS patient experience and satisfaction with effectiveness and symptom improvement with secukinumab, both overall and when compared to their previous treatment. Outcomes were summarised descriptively.

Results: Of 2755 patients screened, 200 patients with AS were eligible for the analysis. The mean (SD) age of survey participants was 34.4 (10.6) years; $60.0 \%$ were male and $66.0 \%$ were white. Equal proportions of patients ( $86.5 \%$ ) had a history of biologic and nonbiologic use, and $98.5 \%$ of patients had previously received treatment for AS; the primary reason for discontinuation of their previous treatment was lack of efficacy $(25.4 \%)$. Most patients $(74.0 \%)$ reported overall improvement in AS symptoms compared with their previous treatment before secukinumab initiation ("a little better," $34.5 \%$; "moderately better," $21.0 \%$; "much better," $18.5 \%$ ). Overall, the majority of patients were satisfied with secukinumab treatment (Abstract SAT0300 - figure 1),. and expressed better treatment experience with secukinumab compared with their previous treatment (Abstract SAT0300 - figure 2)

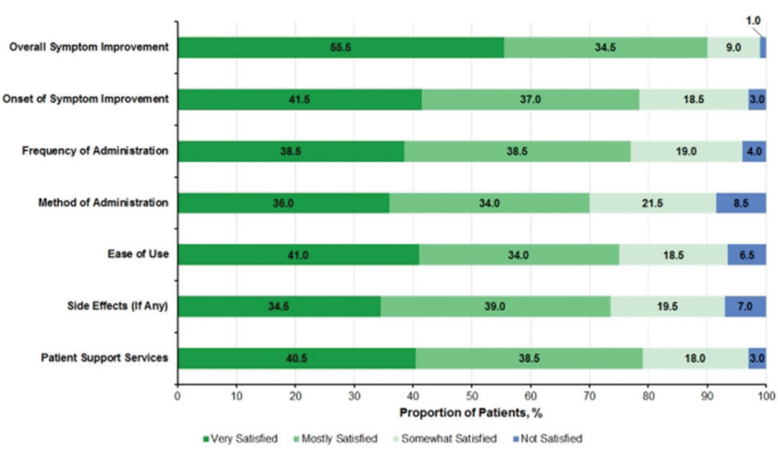

Abstract SAT0300 - Figure 1. Overall satisfaction with secukinumab treatment reported by patients with AS

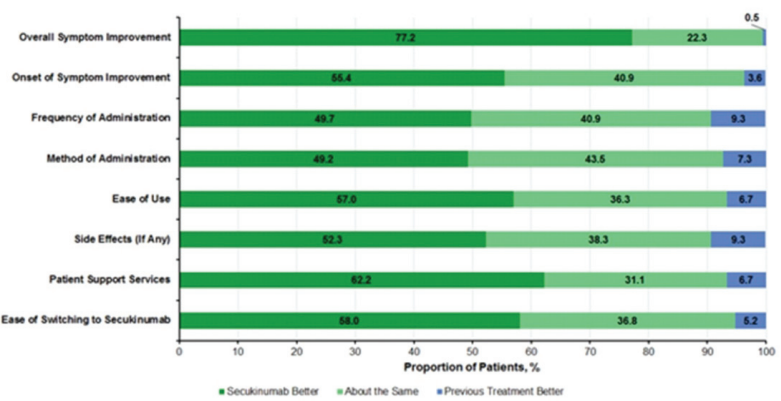

Abstract SAT0300 - Figure 2. Treatment satisfaction with secukinumab for AS compared with previous treatment

Conclusions: Overall, $>90 \%$ of patients with AS who were treated with secukinumab expressed overall satisfaction with their treatment experience, even when compared with their previous treatment. These data provide early insight into the secukinumab treatment experience and satisfaction of US patients with AS.

Acknowledgements: This study was sponsored by Novartis Pharmaceuticals Corporation, East Hanover, NJ.

Disclosure of Interest: M. Magrey Grant/research support from: AbbVie, Consultant for: Janssen, Novartis, UCB, M. Bozyczko: None declared, D. Wolin Employee of: RTI-Health Solutions, M. Mordin Employee of: RTI-Health Solutions, L. Mcleod Employee of: RTI-Health Solutions, E. Davenport Employee of: RTIHealth Solutions, Y. Park Employee of: Novartis Pharmaceuticals Corporation DOI: 10.1136/annrheumdis-2018-eular.1452

\section{SAT0301 \\ SIMILAR EFFICACY OF RHU-TNFR-FC TEPERING AND MAINTENANCE FOR HIP ARTHRITIS IN PATIENTS WITH ANKYLOSING SPONDYLITIS}

Z. Huang, X. Huang, W. Deng, X. Guo, Y. Huang, T. Li. Department of Rheumatology and Immunology, Guangdong Second Provincial General Hospital, Guangzhou, China

Background: Hip involvement is common in patients with ankylosing spondylitis (AS) and eventually leads to functional impairment. Recent studies indicated tumour necrosis factor inhibitors (TNFi) might be an effective therapy for hip arthritis in AS patients. However, the conventional dose of TNFi associates with high cost and increasing risk for adverse effects, so dose reduction is necessary.

Objectives: We compared the efficacy between recombinant human soluble tumour necrosis factor receptor II: IgG Fc fusion protein (Rhu-TNFR-Fc) dose reduction and maintenance in terms of disease activity, function and change of imaging examination. 